\title{
NEW DEVELOPMENTS ON FREE ELECTRON LASERS BASED ON SELF-AMPLIFIED SPONTANEOUS EMISSION
}

\author{
J. Rossbach, DESY, Hamburg, Germany
}

\begin{abstract}
After a brief introduction to the basic concept of Free Electron Lasers operating in the Self-Amplified Spontaneous Emission (SASE) mode, the paper presents the rapid progress achieved during the past 3 years in successfully proving this FEL process at shorter and shorter wavelengths, meanwhile reaching $80 \mathrm{~nm}$. The technical challenges are discussed to be overcome in building FEL facilities for wavelengths much below the visible, and these challenges are compared with the present state-of-the-art. Finally, approved and proposed SASE FEL projects are briefly summarized.
\end{abstract}

\section{INTRODUCTION}

$\mathrm{X}$-ray lasers are expected to open up new and exciting areas of basic and applied research in biology, chemistry and physics. Due to recent progress in accelerator technology the attainment of the long sought-after goal of wide-range tunable laser radiation in the VacuumUltraviolet and X-ray spectral regions is coming close to realization with the construction of Free-Electron Lasers (FEL) based on the principle of Self-Amplified Spontaneous Emission (SASE). In a SASE FEL lasing occurs in a single pass of a relativistic, high-quality electron bunch through a long undulator magnet structure.

The photon wavelength $\lambda_{p h}$ of the first harmonic of FEL radiation is related to the period length $\lambda_{u}$ of a planar undulator by

$$
\lambda_{p h}=\frac{\lambda_{u}}{2 \gamma^{2}}\left(1+\frac{K^{2}}{2}\right),
$$

where $\gamma=E / m_{e} c^{2}$ is the relativistic factor of the electrons, $K=e B_{u} \lambda_{u} / 2 \pi m_{e} c$ the 'undulator parameter' and $B_{u}$ the peak magnetic field in the undulator. Equation (1) exhibits two main advantages of the free-electron laser: the free tunability of the wavelength by changing the electron energy and the possibility to achieve very short photon wavelengths.

For most FELs [1] presently in operation [2], the electron beam quality and the undulator length result in a gain of only a few percent per undulator passage, so that an optical cavity resonator and a synchronized multibunch electron beam have to be used. At very short wavelengths, normal-incidence mirrors of high reflectivity are unavailable. Therefore the generation of an electron beam of extremely high quality in terms of emittance, peak current and energy spread, and a highprecision undulator of sufficient length are essential. Provided the spontaneous radiation from the first part of the undulator overlaps the electron beam, the electromagnetic radiation interacts with the electron bunch leading to a density modulation (micro-bunching) which enhances the power and coherence of radiation. In this "high gain mode" $[3,4,5]$, the radiation power $P(z)$ grows exponentially with the distance $z$ along the undulator

$$
P(z)=P_{0} \cdot A \cdot \exp \left(2 z / L_{g}\right),
$$

where $L_{g}$ is the field gain length, $P_{0}$ the effective input power (see below), and $A$ the input coupling factor $[4,5]$. $A$ is equal to $1 / 9$ in one-dimensional FEL theory with an ideal electron beam.

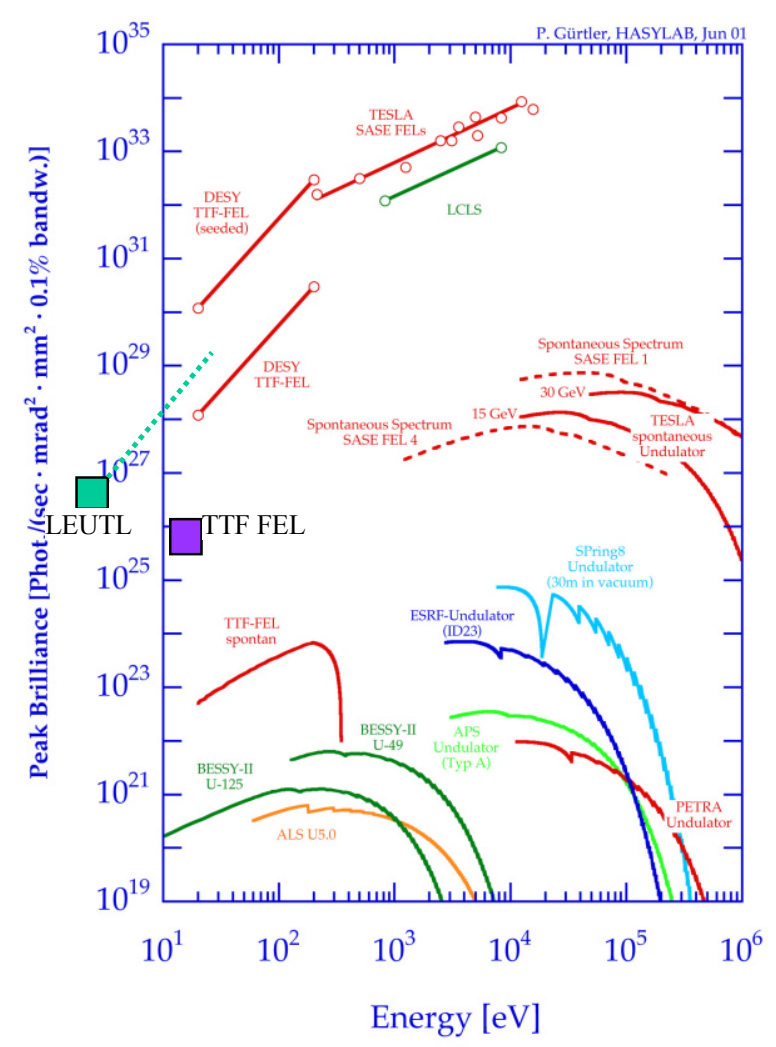

Figure 1: Spectral peak brilliance of X-ray Free Electron Lasers and undulators for spontaneous radiation, in comparison with third-generation synchrotron radiation sources. Results of LEUTL [16,22] and TTF FEL[15] are also shown.

Since the desired wavelength is very short, there is no laser tunable over a wide range to provide the input power $P_{0}$. Instead, the spontaneous undulator radiation from the first part of the undulator is used as an input signal to the downstream part. FELs based on this Self-Amplified- 
Spontaneous-Emission (SASE) principle [6,7] are presently considered the most attractive candidates for delivering extremely brilliant, coherent light with wavelength in the Ångström regime ("XFELs") [8-12,29]. Compared to state-of-the-art synchrotron radiation sources, one expects full transverse coherence, larger average brilliance and, in particular, up to eight or more orders of magnitude larger peak brilliance at pulse lengths of about 200 fs FWHM, see Figure 1.

\section{ACCELERATOR PHYSICS ISSUES}

There are a number of challenges to provide the electron beam of extreme phase space density required for SASE FELs:

- Small momentum width of the order of $10^{-4}$ to ensure all electrons radiate within the bandwidth of the FEL.

- The electron beam orbit within the undulator must be straight in order to guarantee permanent overlap with the radiation field. For XFEL the tolerance is approx. $10 \mu \mathrm{m}$ over $100 \mathrm{~m}$ length.

- To keep the longitudinal velocity spread (eventually destroying the microbunching) within tolerances, the normalized emittance must be approx. $1 \mathrm{mrad} \mathrm{mm}$.

- The FEL saturation length determines the required length of the undulator. To keep this within reasonable limits, the peak current inside the bunch must exceed a few kA. At small beam energy, this is in conflict with small emittance due to space charge forces. Thus, magnetic bunch compression at ultrarelativistic energies has to be performed.

- Coherent synchrotron radiation (CSR) forces generated in the bunch compressor magnets are a serious source of emittance dilution [23,24].

Table 1 summarizes typical XFEL electron beam parameters in comparison with presently achieved performance. It is seen that in all key parameters the extrapolation from proven technology is a factor between 2 and 10 , in contrast to the extrapolation in wavelength of about a factor of 1000 for XFELs. It should also be noted that the TTF injector was not optimised for small emittance. Smaller emittance values have been achieved at other labs, in particular for bunch charges below $1 \mathrm{nC}$. Thus, finding the optimum parameter set in terms of bunch charge, emittance, peak current and tolerances on energy and phase stability is a delicate business.

Table 1: Key electron beam parameters for TESLA XFELs, compared to present performance of the TTF.

\begin{tabular}{|l|c|c|}
\hline & $\begin{array}{l}\text { TTF FEL } \\
\text { now }\end{array}$ & $\begin{array}{l}\text { TESLA FEL } \\
\text { (LCLS similar) }\end{array}$ \\
\hline $\begin{array}{l}\text { Normalized emittance from gun } \\
(\mathrm{Q}=1 \mathrm{nC})\end{array}$ & $3.5 \mathrm{mrad} \mathrm{mm}$ & $0.8 \mathrm{mrad} \mathrm{mm}$ \\
\hline $\begin{array}{l}\text { Norm. emittance at undulator } \\
\text { entrance }\end{array}$ & $8 \mathrm{mrad} \mathrm{mm}$ & $1.6 \mathrm{mrad} \mathrm{mm}$ \\
\hline Beam size in undulator & $100 \mu \mathrm{m}$ & $40 \mu \mathrm{m}$ \\
\hline Bunch length (rms) & $1 \mathrm{ps}$ & $0.1 \mathrm{ps}$ \\
\hline Peak current & $500 \mathrm{~A}$ & $5000 \mathrm{~A}$ \\
\hline Long. emittance $\sigma_{\mathrm{E}} \times \sigma_{1}$ & $100 \mathrm{eV} \mathrm{m}$ & $60 \mathrm{eV} \mathrm{m}$ \\
\hline
\end{tabular}

Design of XFEL injectors needs simultaneous consideration of space charge and CSR effects. An example is the design for the TESLA XEL [25]. It includes a $3^{\text {rd }}$ harmonic RF system to compensate the nonlinear time-dependence of the accelerating field which becomes noticeable if a long bunch (i.e. approx. $2 \mathrm{~mm}$ $\mathrm{rms}$ ) is generated in the RF gun in order to reduce space charge forces, see Fig. 2. A very similar system is under construction for the TTF FEL user facility [26].

Generation of low-emittance, kA peak currents in RF linacs leads, due to the required bunch compression, unavoidably to bunch lengths much smaller than a millimeter. While this is, from the accelerator technology point of view, an unpleasant by-product, it turns out that bunch lengths shorter than $0.1 \mathrm{~mm}$ are an essential benefit for future applications, as the ultra-short radiation pulses allow imaging of fast processes. It is thus requested to push towards even shorter bunch lengths.

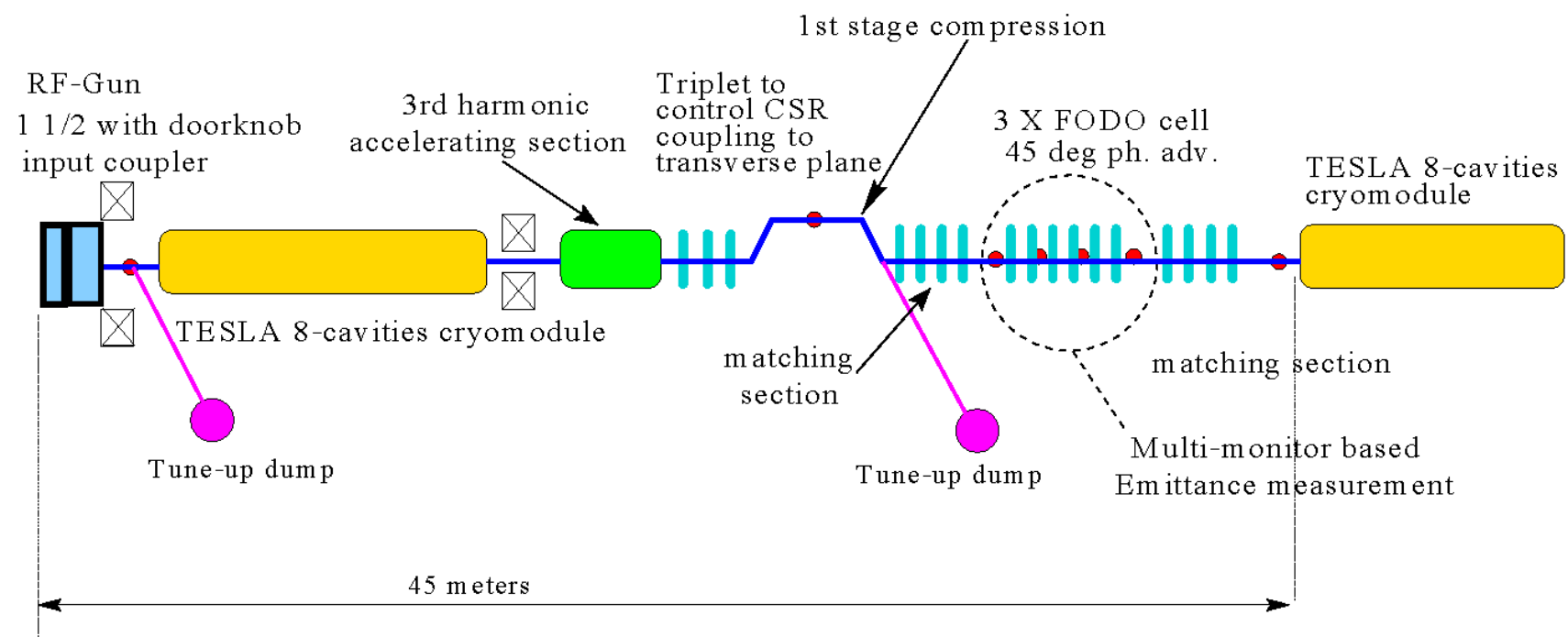

Figure 2: Schematic layout of the TESLA XFEL injector. 
A very important aspect is electron beam diagnostics. For measurement of bunch lengths much smaller than $1 \mathrm{~mm}$, various techniques have been developed. Figure 3 presents the longitudinal bunch profile at TTF FEL measured with two different methods [10,27]. Also, a method streaking the bunch by a transverse mode RF structure has been worked out [28].
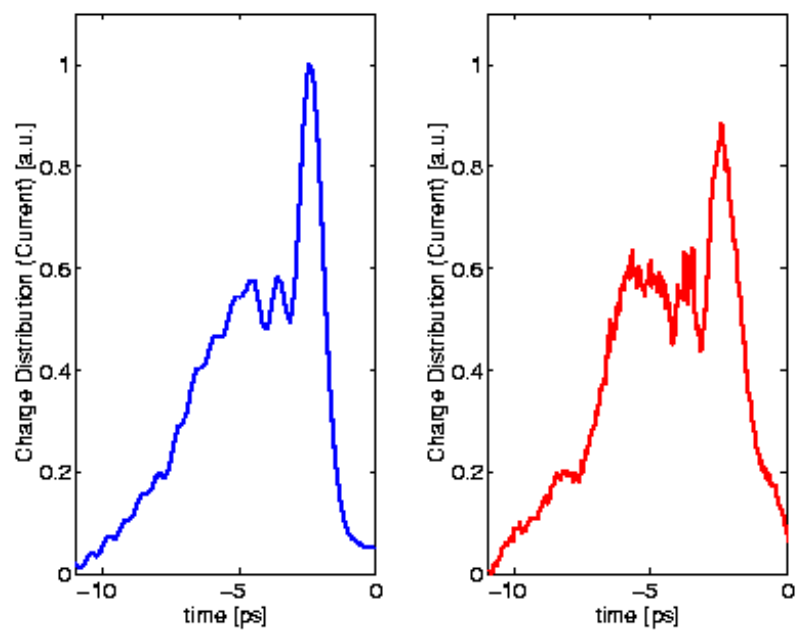

Figure 3: Longitudinal bunch profile measured at TTF FEL with two different methods. Left: Interferometry of coherent synchrotron radiation. Right: Projection from longitudinal phase space tomography.

\section{RECENT SASE TEST RESULTS}

Within the last three years, several important proof-ofprinciple experiments have successfully demonstrated large SASE gain at shorter and shorter wavelength: 12 $\mu \mathrm{m}$ wavelength was achieved 1998 at Los Alamos (here referred to as UCLA/LANL) [13], $530 \mathrm{~nm}$ wavelength was achieved 1999 at Argonne (LEUTL) [14], and 109 $\mathrm{nm}$ wavelength was achieved 2000 at DESY (TTF FEL)[15].

Later, wavelength tuning between 80 and $180 \mathrm{~nm}$ was demonstrated at an FEL gain of approx. $10^{4}$, see Fig. 4.

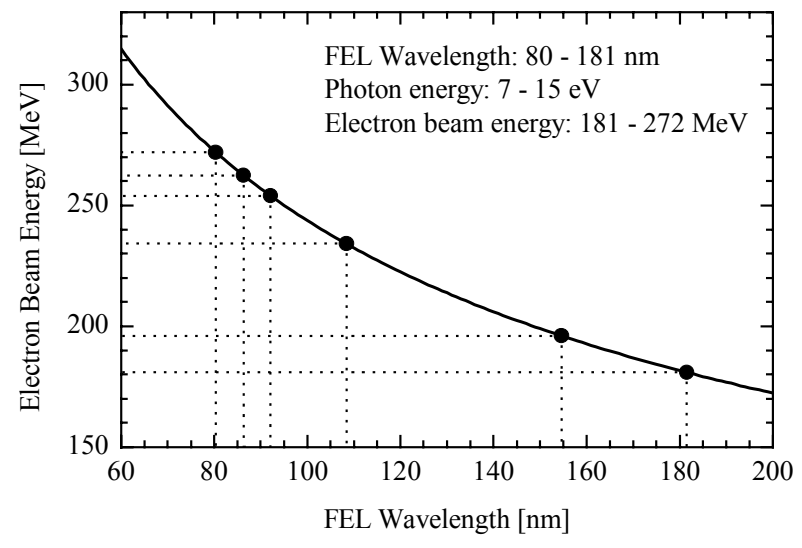

Figure 4: Wavelength tuning demonstrated at the TESLA Test Facility FEL at DESY.
At LEUTL, FEL saturation was observed at 530 and $385 \mathrm{~nm}$ wavelengths [16]. The BNL/LLNL/SLAC/UCLA collaboration has recently demonstrated SASE saturation at $845 \mathrm{~nm}$ at a very short power gain length of only 187 $\mathrm{mm}$ [17]. It is essential that these experiments have been performed at different laboratories and with quite different set-ups. Recent progress on the theoretical understanding includes analysis of 3D-effects [5], the start-up process [18], 3D computer simulation tools [19], generation of high harmonics [20] and photon statistics [21]. These results indicate that the theory to understand and the technology to construct a SASE FEL have attained a rather mature level.

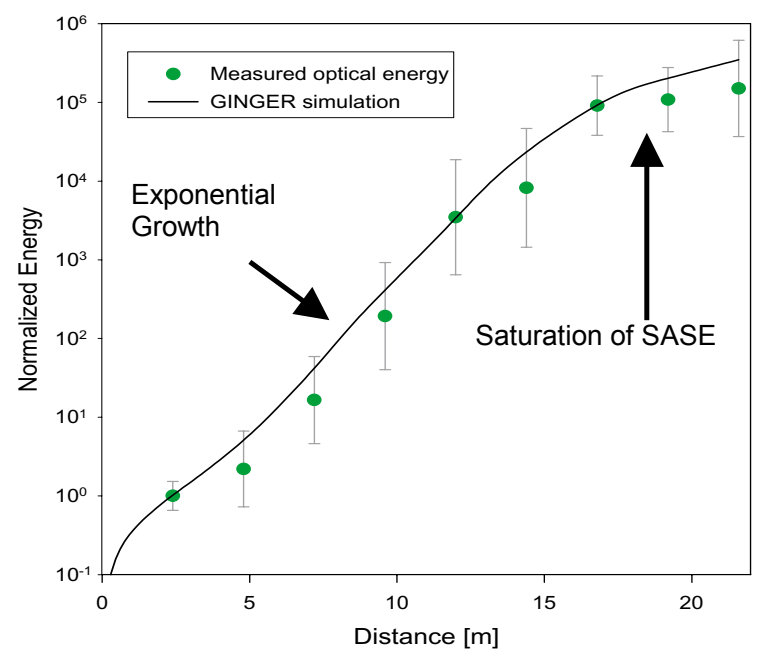

Figure 5: SASE saturation demonstrated at LEUTL/APS (picture taken from [16]). The FEL pulse energy at $530 \mathrm{~nm}$ is plotted vs. distance along the undulator.

1

\section{APPROVED AND PROPOSED PROJECTS}

Several SASE FEL projects are presently approved and under construction, see Table 2. The all aim towards wavelengths far below the visible, since this is the most attractive range for potential users, with no other radiation source of equivalent performance available.

LEUTL will be upgraded in two steps to $120 \mathrm{~nm}$ and $51 \mathrm{~nm}$ wavelength by increasing the beam energy to $457 \mathrm{MeV}$ and $700 \mathrm{MeV}$, respectively [16]. In parallel, the peak current will be increased to $500 \mathrm{~A}$ and the beam emittance reduced, while the undulator length is kept constant at $33 \mathrm{~m}$.

The TESLA Test Facility (TTF) FEL will be upgraded to $1 \mathrm{GeV}$ beam energy. Installing a further stage of bunch compression, 2500 A peak current should be reached, and an improved injector scheme will be realized (see above) to achieve $2 \mathrm{mrad} \mathrm{mm}$ normalized emittance. With $30 \mathrm{~m}$ undulator length, the minimum wavelength $\left(1^{\text {st }}\right.$ harmonic $)$ will be $6 \mathrm{~nm}$. After upgrade, TTF FEL will be turned into 
a SASE FEL user facility. Presently, TTF FEL is the only SASE FEL project based on a superconducting linac. At SPring8 construction of a soft X-ray SASE test facility was started this year. It aims at approx. $5 \mathrm{~nm}$ wavelength. [30].

Table 2: SASE projects under progress and proposed

\begin{tabular}{|c|c|c|}
\hline \multicolumn{3}{|c|}{ SASE FELs under progress } \\
\hline & $\begin{array}{l}\text { Min. } \\
\text { wavelength }\end{array}$ & Schedule \\
\hline APS/LEUTL Phase 2 & $120 \mathrm{~nm}$ & \\
\hline APS/LEUTL Phase 3 & $51 \mathrm{~nm}$ & \\
\hline DESY/TTF FEL Phase 2 & $6 \mathrm{~nm}$ & $2003 / 2004$ \\
\hline SPring8 & $\approx 5 \mathrm{~nm}$ & 2005 \\
\hline \multicolumn{3}{|c|}{ SASE FELs proposed } \\
\hline SLAC/LCLS & $0.15 \mathrm{~nm}$ & $\rightarrow 2006$ \\
\hline DESY/TESLA XFEL & $0.085 \mathrm{~nm}$ & $\rightarrow 2010$ \\
\hline
\end{tabular}

Two SASE FEL user facilities are proposed for operation in the Angstrom wavelength range: LCLS [8,29,31] and TESLA XFEL [10].

The LCLS is based on the part of the SLAC linac not used for the SLAC B-factory. See Figure 6. The proposal aims at operation from 2006 on.

The TESLA XFEL makes common use of part of the superconducting linear accelerator to be constructed for the TESLA linear collider, thus saving major investment costs. Due to the large duty cycle, up to 10000 electron bunches can be accelerated within each RF pulse. Thus, many users can be served in a quasi-simultaneous way by distributing the electron beam, extracted from the linac at the desired energy, to various undulator beamlines, see Figure 7. According to the proposed schedule [10], first operation may begin in 2010.

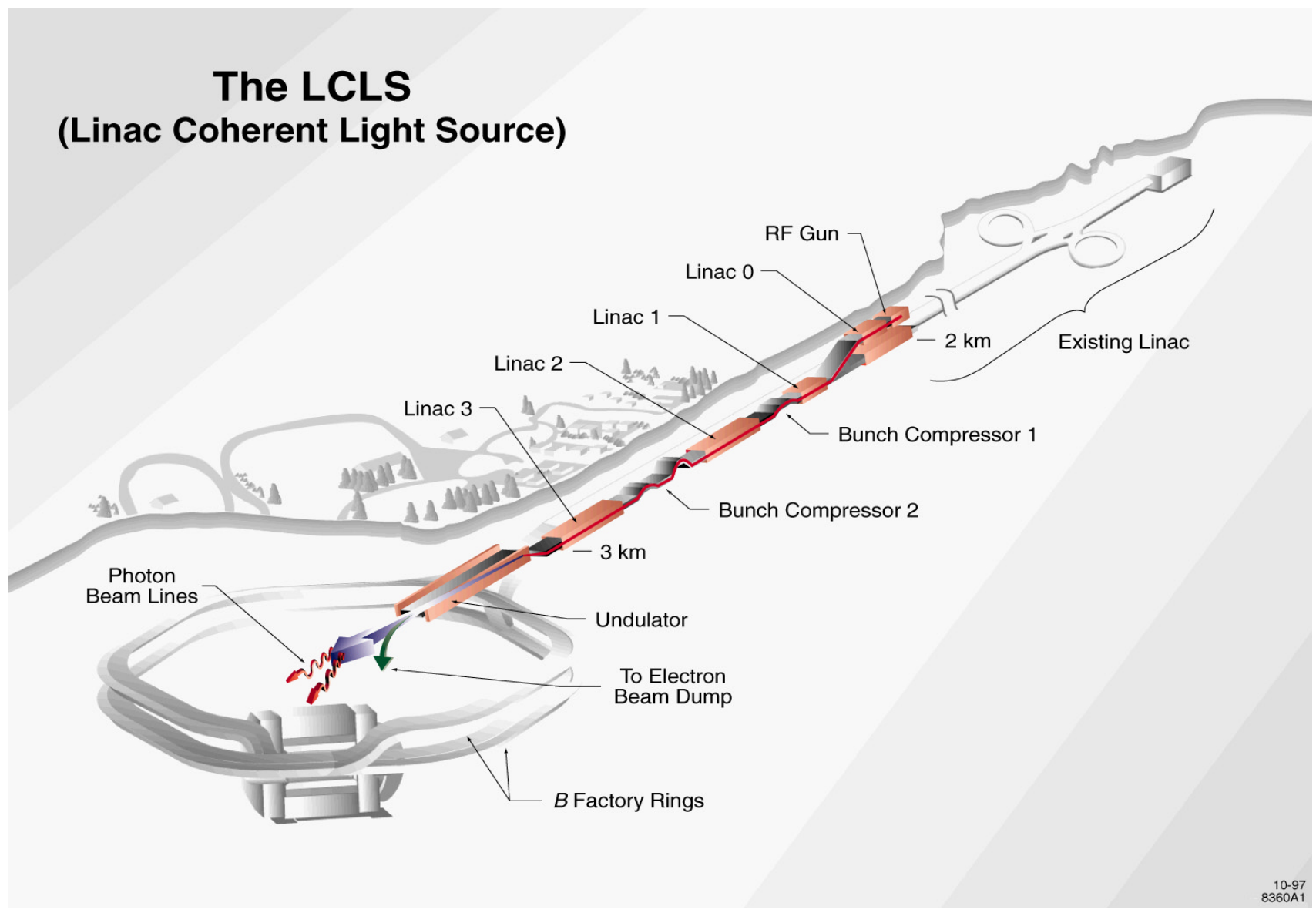

Figure 6: Layout of the LCLS proposed at SLAC. 


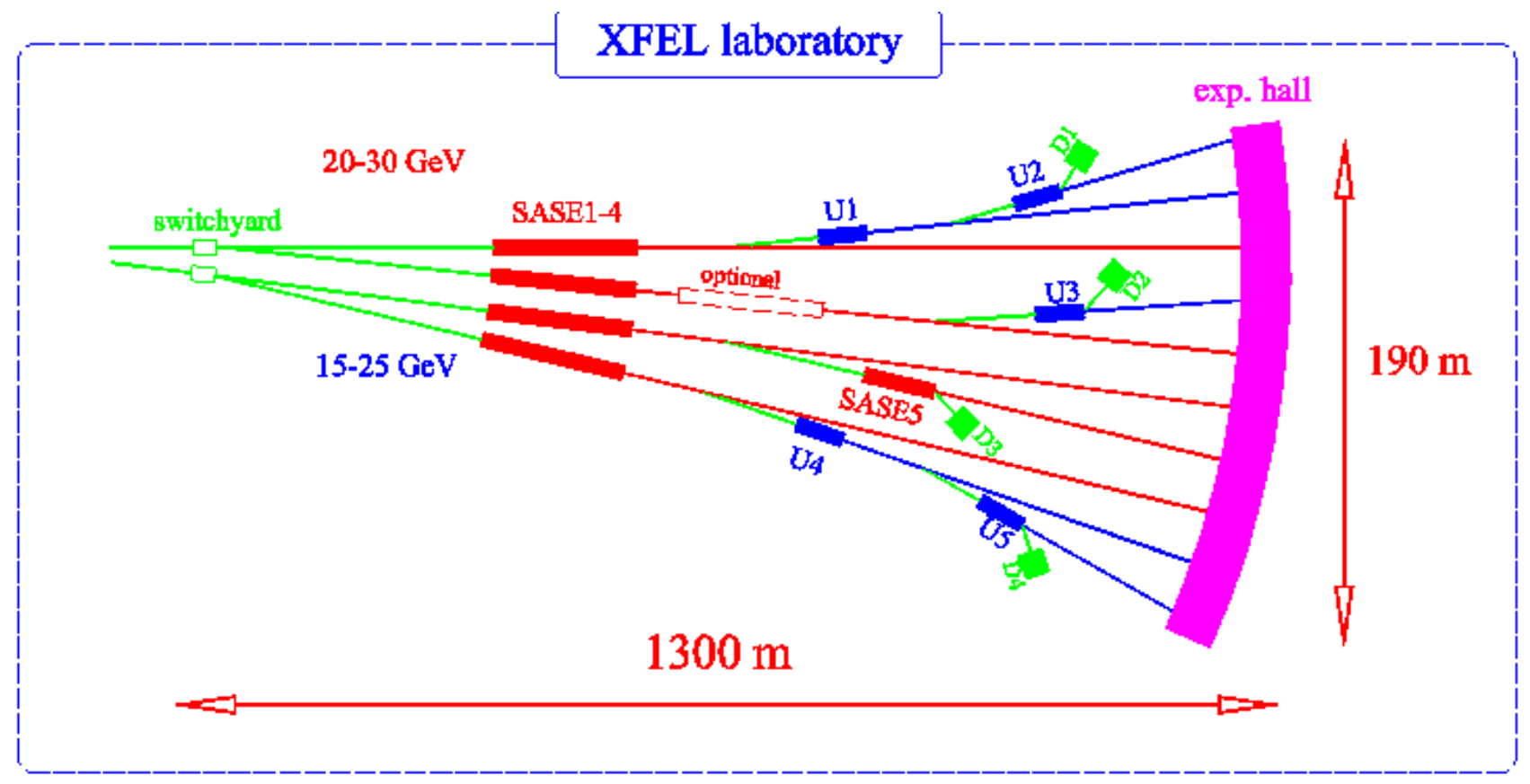

Figure 7: Layout of the beam switchyard of the TESLA XFEL distributing two electron beam lines of different energies to various undulators. SASE1 through SASE5 are FEL undulators while U1 through U5 are undulators for spontaneous radiation. The scheme displays also the long drift lines necessary for the photon beams.

\section{REFERENCES}

[1] J.M. Madey, J. Appl. Phys. 421906 (1971)

[2] W.B. Colson, Nucl. Instr. and Meth. A429 37-40 (1999)

[3] K.J. Kim, Phys. Rev. Lett. 571871 (1986)

[4] S. Krinsky, L.H. Yu, Phys. Rev. A35 3406 (1987)

[5] E.L. Saldin, E.A. Schneidmiller, M.V. Yurkov, "The Physics of Free Electron Lasers", Springer (1999) and references therein

[6] A.M. Kondratenko, E.L. Saldin, Part. Accelerators 10, 207 (1980)

[7] R. Bonifacio, C. Pellegrini, L.M. Narducci, Opt. Commun. 50373 (1984)

[8] H. Winick, et al., Proc. PAC Washington and SLACPUB-6185 (1993)

[9] R. Brinkmann, et al., Nucl. Instr. and Meth. A 393 8692 (1997)

[10] R. Brinkmann, et al. (eds.), DESY 2001-011 and ECFA 2001-209 (2001), http://tesla.desy.de

[11] H.-D. Nuhn, J. Rossbach, Synchrotron Radiation News Vol. 13, No. 1, 18 - 32 (2000)

[12] D. E. Moncton, Proc. Linac Conf., 1048, Argonne (1999)
[13] M. Hogan, et al., Phys. Rev. Lett. 814867 (1998)

[14] S. Milton, et al., Phys. Rev. Lett. 85988 (2000)

[15] J. Andruszkow, et al., Phys. Rev. Lett. 853825 (2000)

[16] S. Milton, et al., this conference

[17] A. Tremain, et al., this conference

[18] Z. Huang, K.J. Kim, Proc. Of 2000 Fel conf,

Durham, to be published in Nucl. Instr. Meth. A

[19] S. Reiche, Nucl. Instr. Meth. A 429, 243 (1999)

[20] L.H. Yu, Science, 289, 932 (2000)

[21] E.L. Saldin, E.A. Schneidmiller, M.V. Yurkov, Opt. Commun. 148, 383 (1998)

[22] Z. Huang, private communication

[23]Ya. S. Derbenev, et al., DESY TESLA-FEL 95-05 (1995)

[24] A. Kabel, M. Dohlus, T. Limberg, Nucl. Instr.

Meth. A 455185 (2000)

[25] P. Piot, et al., DESY TESLA-FEL 01-03 (2001)

[26] T. Åberg, et al., DESY TESLA-FEL 95-03 (1995)

[27] M. Hüning, to be published

[28] P. Krejcik et. al., this conference

[29] J. Arthur, et al., SLAC-R-521 (1998)

[30] T. Nakamura, private communication

[31] P. Emma, this conference 